\title{
To fire or not to fire: origin activation in Saccharomyces cerevisiae ribosomal DNA
}

\author{
Andreas S. Ivessa and Virginia A. Zakian ${ }^{1}$ \\ Department of Molecular Biology, Princeton University, Princeton, New Jersey 08544-1014, USA
}

Duplication of eukaryotic chromosomes begins from multiple sites called origins of DNA replication, with replication typically proceeding bidirectionally from each origin. The yeast Saccharomyces cerevisiae is the only eukaryote in which there is a detailed understanding of the sites used for initiation of chromosomal DNA replication. In yeast, these sites are called ARSs (autonomously replicating sequences) because they were initially identified by their ability to confer high-frequency transformation and self-replication on plasmids introduced into cells (Stinchcomb et al. 1979). By these criteria, there are 200-400 ARS elements in the yeast genome. Using two-dimensional (2D) gel electrophoresis, some-but not all-ARSs can be shown to be origins of replication when situated at their normal chromosomal loci (for review, see Newlon and Theis 2002).

ARS elements are relatively small, $\sim 100-150 \mathrm{bp}$, and consist of one or more copies of an essential 11-bp-long AT-rich ARS consensus sequence, as well as several other less conserved elements. The ARS consensus sequence is the binding site for the multi-subunit origin recognition complex (ORC), which binds constitutively throughout the cell cycle and is essential for initiation (for review, see Bell and Dutta 2002). Several proteins are recruited to the ARS during the $G_{1}$ phase of the cell cycle to form a prereplicative complex, including the multisubunit minichromosome maintenance (MCM) complex, which has ATPase and helicase activity and is needed for both initiation and fork progression.

Recently, two laboratories used genome-wide microarray analysis to determine the positions of replication origins in the Saccharomyces genome (Raghuraman et al. 2001; Wyrick et al. 2001). Raghuraman and colleagues (2001) determined the time of replication of each segment of the 16 yeast chromosomes. They used this information to identify 332 sites where bidirectional replication begins, although many of these origins are not active in every cell cycle. Wyrick and colleagues (2001) used a complementary approach: They determined sites

${ }^{1}$ Corresponding author.

E-MAIL vzakian@molbio.princeton.edu; FAX (609) 258-1701.

Article and publication are at http://www.genesdev.org/cgi/doi/10.1101/ gad.1033702. of binding of both the ORC and MCM complexes, finding 429 sites that are bound by both complexes. There is high but not complete overlap between the sites identified as origins by the two methods, suggesting that although ORC and MCM binding is highly correlated with origin activity, this binding is not sufficient and, in rare cases, may even be unnecessary for origin activity.

Despite the wealth of information about initiation of DNA replication in Saccharomyces, it is still uncertain what determines whether a given yeast ARS acts as an origin or how the efficiency and time of activation of active origins is regulated (for review, see Pasero and Schwob 2000). Existing data suggest that both chromosomal context and chromatin structure affect origin activity. For example, if an origin is near a telomere, activation of this origin occurs late in S phase (Ferguson et al. 1991). If the same origin is placed on a plasmid, initiation occurs early in the cell cycle. However, linearization of this plasmid by addition of telomere sequences to both ends causes the origin to again be activated late (Ferguson and Fangman 1992). These data demonstrate that telomeres exert a position effect on the activation of nearby origins, a function reminiscent of their inhibitory effect on transcription of nearby genes (for review, see Tham and Zakian 2002).

In some cases, chromosomal context actually prevents rather than simply delaying origin activation. For example, ARSs near $H M L$, one of two transcriptionally quiescent mating type loci on chromosome III, and some origins in subtelomeric regions (Newlon et al. 1993; Ivessa et al. 2002) are typically inactive (Fig. 1). Although $A R S 301$, which overlaps the location of the E silencer at $H M L$, is not active in its normal chromosomal context, if it is moved away from $H M L$ to the position of and replacing ARS305, the first active origin on the left arm of chromosome III, ARS301 becomes active (Fig. 1; Vujcic et al. 1999). Further, the probability that a normally inactive ARS will become active increases the more time it takes a fork emanating from an active origin to reach the silent ARS. For example, in the $H M L$ region, if the active origin ARS305 is deleted, the ARS cluster 302, 303, 320 becomes active (Fig. 1). If the two active origins ARS305 and ARS306 are both absent, both ARS301 (at the E si- 


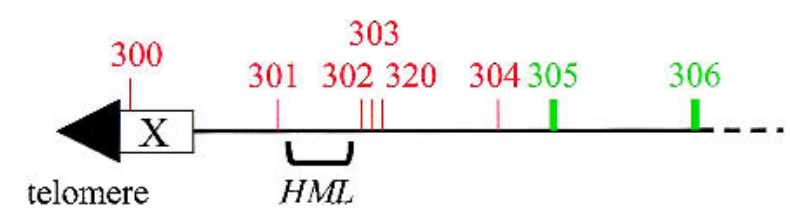

Figure 1. The left arm of chromosome III in yeast. An $80-\mathrm{kbp}$ region adjacent to the left telomere of chromosome III is shown (figure is not drawn to scale). This region contains eight ARSs (autonomously replicating sequences), as defined by their ability to promote autonomous replication of plasmid DNA. However, only ARS305 and ARS306 are normally active as origins of DNA replication. Active origins are in green; inactive origins are in red. The black arrow head represents $\sim 300 \mathrm{bp}$ of $\mathrm{C}_{1-3} \mathrm{~A} /$ $\mathrm{TG}_{1-3}$ telomeric DNA. $\mathrm{X}$ is a subtelomeric, middle repetitive sequence that is found at virtually all yeast telomeres. $H M L$ is one of two silent mating type loci. ARS301 is part of the E silencer, and $A R S 302$ is part of the I silencer. $H M L$ is, respectively, $25 \mathrm{~kb}$ from ARS305 and $\sim 60 \mathrm{~kb}$ from ARS306.

lencer) and the $A R S$ cluster containing 302 (at the I silencer), 303, and 320 become active, although in both cases activation is inefficient (Vujcic et al. 1999). Likewise, if replication fork progression is slowed by the absence of the Rrm3p DNA helicase, normally silent ARSs in the subtelomeric $\mathrm{X}$ and $\mathrm{Y}^{\prime}$ elements, have an increased probability of firing (Ivessa et al. 2002).

Chromatin structure also affects origin activation. Transcriptional silencing at both telomeres and the silent mating type loci requires the silencing proteins Sir2p, Sir3p, and Sir4p (for review, see Grunstein 1998; Gartenberg 2000). The chromatin of transcriptionally silent regions has several features that distinguish it from transcriptionally active chromatin. For example, histones in silent regions are hypoacetylated compared with histones in most other regions of the genome. At both telomeres and the silent mating type loci, the structural changes characteristic of silent chromatin are lost in strains lacking any one of the three Sir proteins. Likewise, Sir3p influences the probability of activation of some origins in transcriptionally silent regions. When Sir3p is absent, origins in the subtelomeric $\mathrm{Y}^{\prime}$ elements are activated earlier in the $S$ phase than in wild-type cells, whereas a normally inactive ARS in a subtelomeric $\mathrm{X}$ element becomes active in some cells (Stevenson and Gottschling 1999). However, neither the time of firing of the late-replicating origin ARS501, which is about 25 kbp from a telomere (Stevenson and Gottschling 1999), nor the activation of the normally silent ARSs at the $H M L$ silent mating type locus, is altered in the absence of Sir3p (Vujcic et al. 1999). Thus, regional loss of the characteristic chromatin features associated with transcriptional repression is not sufficient to relieve the inhibitory effect of these regions on DNA replication.

Much less is known in other eukaryotes about the sites used for initiation of DNA replication. Although ARSs have been identified by the plasmid assay in fission yeast, these ARSs are much larger than Saccharomyces $A R S$ s, and no one essential $A R S$ consensus sequence has been identified (Clyne and Kelly 1995). In multicellular eukaryotes, origin usage is developmentally regulated, with many more origins used early in development when the rate of DNA replication is high. Although ORC binding sites have been identified in fission yeast (Chuang et al. 2002) and flies (for review, see Gerbi and Bielinsky 2002), the only common theme so far identified is that these binding sites are AT-rich. In Saccharomyces, most origins are located in nontranscribed intergenic regions, which in yeast are small. In contrast, chromosomes in multicellular organisms have a far higher fraction of nontranscribed DNA. It has been proposed that the high density of genes in Saccharomyces imposes a requirement for sequence-specific initiation to ensure that initiation occurs in intergenic regions, whereas the higher fraction of intergenic DNA in multicellular eukaryotes obviates this requirement (Brewer 1994). These considerations suggest that chromosomal context and chromatin structure are likely to have an even greater role in origin specification in higher cells than they do in yeast.

The Saccharomyces rDNA provides a model system for how organisms selectively activate replication origins. In yeast, there are 100-200 identical copies of the 9.1-kb rDNA repeat, organized in a single large tandem array on chromosome XII (Fig. 2). Each repeat encodes both the 35S rRNA, the precursor to $18 \mathrm{~S}$ and $25 \mathrm{~S}$ rRNAs, and $5 \mathrm{~S}$ rRNA. The nontranscribed spacer of each repeat contains two cis-acting sequences that affect DNA replication, an ARS (Skryabin et al. 1984) and a replication fork barrier (RFB; Fig. 2; Brewer and Fangman 1988; Linskens and Huberman 1988). The pattern of rDNA replication was determined using 2D gel electrophoresis (Fig. 2; Brewer and Fangman 1988; Linskens and Huberman 1988). Replication begins and proceeds bidirectionally from the ARS. Despite their apparent sequence identity, only about $20 \%$ of the ARSs in the rDNA are active in any given S phase (Saffer and Miller 1986; Brewer and Fangman 1988; Linskens and Huberman 1988). Activation of origins in only a subset of rDNA repeats helps explain why rDNA replication occurs throughout the entire S phase (Brewer et al. 1980). Although replication is initially bidirectional, when the leftward-moving fork encounters the RFB, it stops. The RFB is a highly efficient but polar block to fork progression: Only forks that approach the RFB from the right are affected, whereas rightward-moving forks proceed unimpeded past each RFB they encounter. At the end of rDNA replication, in the subset of repeats that had an active origin, rightwardmoving forks converge on forks stalled at the RFB. As a result of this unusual mode of replication, replication of the rDNA is mostly unidirectional, a replication mode that is distinct from that of the rest of the genome. Cisacting sequences that impede replication fork progression in rDNA have also been described in fission yeast and multicellular organisms, indicating that this unusual rDNA replication mechanism is widespread amongst eukaryotes (Gerber et al. 1997; Lopez-estrano et al. 1998; Sanchez et al. 1998).

Although it is clear from the 2D gel analysis that only a subset of rDNA ARSs is active, this approach can not determine the distribution of active versus inactive origins. Moreover, because the rDNA repeats are identical, 


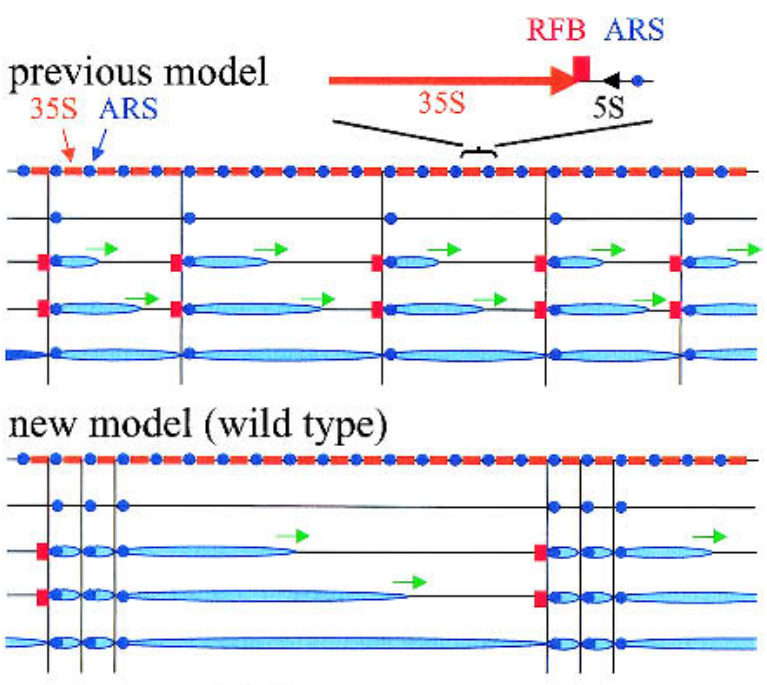

new model (sir2)

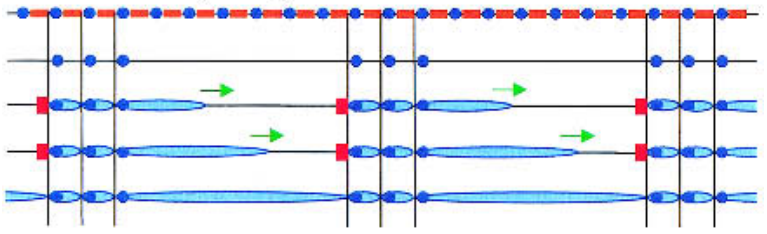

Figure 2. Replication of Saccharomyces ribosomal DNA. rDNA from yeast is arranged as a tandem array of 100-200 identical 9.1-kb repeats. Each repeat contains two transcription units [35S rRNA (orange) and 5S rRNA (black)] in opposite orientation to each other, a putative origin of DNA replication (ARS, blue circles), and a site that confers unidirectional replication to the array, the replication fork barrier (RFB, red rectangle). The elongated light-blue ovals represent the elongating replication forks. After initiation, leftward-moving forks stop at the RFB, whereas rightward-moving forks continue until they converge with a fork arrested at the RFB. For simplicity, the RFB is only shown in repeats where it impedes fork progression. Based on previous observations, only $\sim 20 \%$ of ARSs are active as origins in a given $\mathrm{S}$ phase. These data led to an earlier model in which repeats with active origins are spaced relatively uniformly throughout the rDNA. Using dynamic molecular combing, Pasero et al. (2002) show that initiation of rDNA replication actually occurs by activation of two to three origins in adjacent repeats, with regions containing active origins being separated from each other by large gaps that lack initiation events. The sizes of these origin-less gaps are decreased in a sir2 strain, indicating that Sir2p suppresses initiation of DNA replication in rDNA.

this question can also not be addressed by the genomewide approaches described earlier. Rather, this issue can only be tackled using methods that can examine replication throughout an intact rDNA array, a feat that is technically challenging given the 1 - to $2-\mathrm{Mb}$ size of the array. Earlier attempts at addressing this question used electron microscopy (Saffer and Miller 1986) or sucrose gradients (Walmsley et al. 1984) to examine replication of large segments of yeast rDNA. Although both studies suggested that initiation of replication often occurs in adjacent rDNA repeat units, neither approach is precise enough to provide an array-wide view of the distribution of origins or to determine if the pattern of origin use is maintained from one generation to the next.

In this issue of Genes and Development, Pasero and colleagues (2002) use a novel and elegant approach to examine rDNA replication in Saccharomyces at the level of single chromosomal DNA molecules. The authors first arrest cells in $G_{1}$ phase and then release them into $S$ phase in the presence of bromodeoxyuridine (BrdU). Because yeast does not encode a thymidine kinase (TK) gene, which is required for the incorporation of BrdU into DNA, the authors use a yeast strain that constitutively expresses the herpes simplex virus TK gene (Lengronne et al. 2001). After a brief incubation in BrdU, cells are chased with deoxythymidine, such that only early-replicating, hence origin-proximal, DNA is BrdU labeled. To facilitate analysis, total genomic DNA is digested with restriction enzymes that do not cut within rDNA repeats, releasing the rDNA locus as a $\sim 1.5-\mathrm{Mb}$ fragment and reducing most of the genomic DNA to small pieces. The DNA is then analyzed with a technique called dynamic molecular combing (DMC), which allows a uniform stretching of DNA on a glass support (for review, see Caburet et al. 2002). The end result is that all DNA molecules are aligned relative to each other in parallel orientation. Individual rDNA repeat units are visualized by hybridization to $35 \mathrm{~S}$ rDNA (red spots; Pasero et al. 2002, Fig. 1). Repeats that incorporate BrdU during early $S$ phase are inferred to have an active replication origin and are detected using antibodies to BrdU (green regions; Pasero et al. Fig. 1).

This analysis clearly reveals that active origins in yeast rDNA are clustered: Activation occurs in two to three adjacent repeats with gaps of differing lengths between actively replicating units (Fig. 2). Although the spacing between regions with active origins is quite variable, perhaps the most striking aspect of the replication pattern is the abundance of long stretches without active origins: Almost one third of the gaps between initiation sites are $>60 \mathrm{~kb}$, indicating $\geq 7$ adjacent repeats in which the rDNA ARS is not active.

The methods used in this paper provide perhaps a unique opportunity to determine if the pattern of initiation site usage in the rDNA is inherited. The question the investigators wish to address is whether or not the $20 \%$ of rDNA ARSs that are active as origins in a given cell are also used in the progeny of that cell. The investigators use a simple but elegant variation of the previously described methods. To align different arrays relative to each other, the investigators hybridize the DMCstretched DNA to a probe that detects a long stretch of DNA immediately upstream of the rDNA array while simultaneously detecting individual repeats using the probe for the $35 \mathrm{~S}$ rDNA and origin proximal DNA using anti BrdU antibodies. Using this approach, they find that the pattern of initiation sites is not identical among different rDNA arrays, suggesting that these patterns are not inherited. However, there are limits to this analysis: For example, the initiation pattern might be largely inherited but subject to slow and/or continuous change in a subset of the repeats. 
In exponentially growing yeast cells, only about half of the rDNA repeats are transcribed, and this proportion varies in response to growth conditions (Dammann et al. 1993). Thus, paradoxically, given its high overall rate of transcription, rDNA transcription is repressed in many repeats. Likewise, transcription of RNA polymerase IItranscribed genes that are inserted into the rDNA is reversibly repressed, a phenomenon similar to the transcriptional silencing seen at telomeres and the silent mating type loci. This rDNA silencing requires Sir2p, but not Sir3p or Sir4p (Bryk et al. 1997; Smith and Boeke 1997). Sir2p is a NAD ${ }^{+}$-dependent histone deacetylase that removes acetylated lysines from histones $\mathrm{H} 3$ and H4 (Imai et al. 2000; Landry et al. 2000; Smith et al. 2000). Point mutations that eliminate the deacetylase activity of Sir2p also eliminate its in vivo silencing at telomeres, the silent mating type loci, and the rDNA, arguing that Sir2p likely promotes transcriptional silencing by helping maintain the hypoacetylation characteristic of transcriptionally repressed chromatin (Imai et al. 2000). In addition to its effects on rDNA transcription, Sir2p also specifically inhibits recombination in the rDNA (Gottlieb and Esposito 1989), and this inhibition is also lost in sir2 strains that lack the histone deacetylase activity of Sir2p (Imai et al. 2000).

Electron microscopy reveals that transcription and replication occur on the same rDNA molecules (Saffer and Miller 1986). Moreover, replication and transcription appear to be linked, as initiation of DNA replication invariably occurs in rDNA repeats that are immediately downstream of transcriptionally active rDNA genes (Muller et al. 2000). Given its effects on rDNA transcription and recombination, the investigators tested if Sir2p also affects origin usage within the rDNA. Using the DMC technique, they found that the number of clusters of active origins increases almost twofold in a sir2 strain, a result confirmed by the more standard $2 \mathrm{D}$ gel technique. This result suggests that in wild-type cells, Sir2p suppresses the initiation of DNA replication within rDNA (Fig. 2). Although the number of initiation clusters is increased in the absence of Sir $2 p$, initiation events are still clustered, indicating that this feature of rDNA replication is not Sir2p dependent.

How might Sir2p affect rDNA replication? Sir2p could affect origin firing directly because by the criterion of chromatin immunoprecipitation, it is physically associated in the vicinity of the rDNA ARS (Gotta et al. 1997; Hoppe et al. 2002). For example, Sir2p might affect the acetylation status of a critical replication protein. Alternatively, by virtue of its histone deacetylase activity, Sir2p might influence the higher-order chromatin structure of rDNA. In this model, absence of Sir2p-mediated deacetylation causes an unfolding of rDNA chromatin, making it more accessible to replication, recombination, and transcription. Support for this hypothesis comes from the demonstration that rDNA chromatin structure is altered in a sir2 strain: sir2 rDNA chromatin is more accessible to micrococcal nuclease digestion at specific sites in the nontranscribed spacer, including the region containing the $A R S$ (Fritze et al. 1997). Both models pre- dict that the deacetylase activity of Sir2p will be critical for its effects on rDNA replication, a prediction that can be tested easily with the DMC method. Alternatively, the ADP-ribosyltranferase activity of Sir2p might inhibit origin usage, although this activity is relatively weak (Tanny et al. 1999). Finally, because Sir2p is rDNA associated, it might have a structural role in rDNA chromatin that affects rDNA replication.

It is increasingly evident that there are links between DNA replication and transcriptional silencing. For example, physical and functional associations between ORC and silencing proteins have been reported both in Saccharomyces (Triolo and Sternglanz 1996; Fox et al. 1997) and in multicellular organisms (Pak et al. 1997; Shareef et al. 2001). Given the results reported in Pasero et al. (2002), it would not be surprising if the distribution of active transcription units in the yeast rDNA is also regulated by Sir2p. Because silent chromatin is hypoacetylated in diverse organisms, the Sir2p-mediated repression of origin activation seen in yeast rDNA may reflect a general connection between histone acetylation status and control of origin firing. In addition to the Sir2p family of $\mathrm{NAD}^{+}$-dependent histone deacetylases, Saccharomyces encodes at least five other histone deacetylases, two of which act preferentially within rDNA and hence might act in concert with Sir2p to affect rDNA replication (Robyr et al. 2002). Histone acetylation might also regulate origin firing in more conventionally transcribed regions of the genome, a possibility that is currently being tested in several labs. These experiments will be greatly aided by genome-wide analyses that identify the specific regions affected by individual histone deacetylases (Kurdistani et al. 2002; Robyr et al. 2002).

\section{Acknowledgments}

Work in the Zakian lab is supported by grants from the National Institutes of Health. A.S.I. is supported by a grant from the Leukemia and Lymphoma Society.

\section{References}

Bell, S.P. and Dutta, A. 2002. DNA replication in eukaryotic cells. Annu. Rev. Biochem. 71: 333-374.

Brewer, B.J. 1994. Intergenic DNA and the sequence requirements for replication initiation in eukaryotes. Curr. Opin. Genet. Dev. 4: 196-202.

Brewer, B.J. and Fangman, W.L. 1988. A replication fork barrier at the $3^{\prime}$ end of yeast ribosomal RNA genes. Cell 55: 637643.

Brewer, B.J., Zakian, V.A., and Fangman, W.L. 1980. Replication and meiotic transmission of yeast ribosomal RNA genes. Proc. Nat1. Acad. Sci. 77: 6739-6743.

Bryk, M., Banerjee, M., Murphy, M., Knudsen, K.E., Garfinkel, D.J., and Curcio, M.J. 1997. Transcriptional silencing of Ty1 elements in the RDN1 locus of yeast. Genes \& Dev. 11: 255269.

Caburet, S., Conti, C., and Bensimon, A. 2002. Combing the genome for genomic instability. Trends Biotechnol. 20: 344350.

Chuang, R.Y., Chretien, L., Dai, J., and Kelly, T.J. 2002. Purifi- 
cation and characterization of the Schizosaccharomyces pombe origin recognition complex: Interaction with origin DNA and Cdc18 protein. J. Biol. Chem. 277: 16920-16927.

Clyne, R.K. and Kelly, T.J. 1995. Genetic analysis of an ARS element from the fission yeast Schizosaccharomyces pombe. EMBO J. 14: 6348-6357.

Dammann, R., Lucchini, R., Koller, T., and Sogo, J.M. 1993. Chromatin structures and transcription of rDNA in yeast Saccharomyces cerevisiae. Nucleic Acids Res. 21:23312338.

Ferguson, B.M. and Fangman, W.L. 1992. A position effect on the time of replication origin activation in yeast. Cell 68: 333-339.

Ferguson, B.M., Brewer, B.J., Reynolds, A.E., and Fangman, W.L. 1991. A yeast origin of replication is activated late in $S$ phase. Cell 65: 507-515.

Fox, C.A., Ehrenhofer-Murray, A.E., Loo, S., and Rine, J. 1997. The origin recognition complex, SIR1, and the S phase requirement for silencing. Science 276: 1547-1551.

Fritze, C.E., Verschueren, K., Strich, R., and Easton Esposito, R. 1997. Direct evidence for SIR2 modulation of chromatin structure in yeast rDNA. EMBO J. 16: 6495-6509.

Gartenberg, M.R. 2000. The Sir proteins of Saccharomyces cerevisiae: Mediators of transcriptional silencing and much more. Curr. Opin. Microbiol. 3: 132-137.

Gerber, J.K., Gogel, E., Berger, C., Wallisch, M., Muller, F., Grummt, I., and Grummt, F. 1997. Termination of mammalian rDNA replication: Polar arrest of replication fork movement by transcription termination factor TTF-I. Cell 90: 559-567.

Gerbi, S.A. and Bielinsky, A.K. 2002. DNA replication and chromatin. Curr. Opin. Genet. Dev. 12: 243-248.

Gotta, M., Strahl-Bolsinger, S., Renauld, H., Laroche, T., Kennedy, B.K., Grunstein, M., and Gasser, S.M. 1997. Localization of Sir2p: The nucleolus as a compartment for silent information regulators. EMBO J. 16: 3243-3255.

Gottlieb, S. and Esposito, R.E. 1989. A new role for a yeast transcriptional silencer gene, SIR2, in regulation of recombination in ribosomal DNA. Cell 56: 771-776.

Grunstein, M. 1998. Yeast heterochromatin: Regulation of its assembly and inheritance by histones. Cell 93: 325-328.

Hoppe, G.J., Tanny, J.C., Rudner, A.D., Gerber, S.A., Danaie, S., Gygi, S.P., and Moazed, D. 2002. Steps in assembly of silent chromatin in yeast: Sir3-independent binding of a Sir2/Sir4 complex to silencers and role for Sir2-dependent deacetylation. Mol. Cell Biol. 22: 4167-4180.

Imai, S., Armstrong, C.M., Kaeberlein, M., and Guarente, L. 2000. Transcriptional silencing and longevity protein Sir2 is an NAD- dependent histone deacetylase. Nature 403: 795800.

Ivessa, A.S., Zhou, J.-Q. Schulz, V.P., Monson, E.M., and Zakian, V.A. 2002. Saccharomyces Rrm3p, a 5' to 3' DNA helicase that promotes replication fork progression through telomeric and sub-telomeric DNA. Genes \& Dev. 16: 13831396.

Kurdistani, S.K., Robyr, D., Tavazoie, S., and Grunstein, M. 2002. Genome-wide binding map of the RPD3 histone deacetylase in yeast. Nat. Genet. 31: 248-254.

Landry, J., Sutton, A., Tafrov, S.T., Heller, R.C., Stebbins, J., Pillus, L., and Sternglanz, R. 2000. The silencing protein SIR2 and its homologs are NAD-dependent protein deacetylases. Proc. Nat1. Acad. Sci. 97: 5807-5811.

Lengronne, A., Pasero, P., Bensimon, A., and Schwob, E. 2001. Monitoring $S$ phase progression globally and locally using BrdU incorporation in $\mathrm{TK}(+)$ yeast strains. Nucleic Acids
Res. 29: 1433-1442.

Linskens, M.H.K. and Huberman, J.A. 1988. Organization of replication of ribosomal DNA in Saccharomyces cerevisiae. Mol. Cell Biol. 8: 4927-4935.

Lopez-estrano, C., Schvartzman, J.B., Krimer, D.B., and Hernandez, P. 1998. Co-localization of polar replication fork barriers and rRNA transcription terminators in mouse rDNA. J. Mol. Biol. 277: 249-256.

Muller, M., Lucchini, R., and Sogo, J.M. 2000. Replication of yeast rDNA initiates downstream of transcriptionally active genes. Mol. Cell 5: 767-777.

Newlon, C.S. and Theis, J.F. 2002. DNA replication joins the revolution: Whole-genome views of DNA replication in budding yeast. Bioessays 24: 300-304.

Newlon, C.S., Collins, I., Dershowitz, A., Deshpande, A.M., Greenfeder, S.A., Ong, L.Y., and Theis, J.F. 1993. Analysis of replication origin function on chromosome III of Saccharomyces cerevisiae. Cold Spring Harbor Symp. Quant. Biol. 58: 415-423.

Pak, D.T., Pflumm, M., Chesnokov, I., Huang, D.W., Kellum, R., Marr, J., Romanowski, P., and Botchan, M.R. 1997. Association of the origin recognition complex with heterochromatin and HP1 in higher eukaryotes. Cell 91: 311-323.

Pasero, P. and Schwob, E. 2000. Think global, act local: How to regulate $S$ phase from individual replication origins. Curr. Opin. Genet. Dev. 10: 178-186.

Pasero, P., Bensimon, A., and Schwob, E. 2002. Single-molecule analysis reveals clustering and epigenetic regulation of replication origins at the yeast rDNA locus. Genes \& Dev., this issue.

Raghuraman, M.K., Winzeler, E.A., Collingwood, D., Hunt, S., Wodicka, L., Conway, A., Lockhart, D.J., Davis, R.W., Brewer, B.J., and Fangman, W.L. 2001. Replication dynamics of the yeast genome. Science 294: 115-121.

Robyr, D., Suka, Y., Xenarios, I., Kurdistani, S.K., Wang, A., Suka, N., and Grunstein, M. 2002. Microarray deacetylation maps determine genome-wide functions for yeast histone deacetylases. Cell 109: 437-446.

Saffer, L.D. and Miller, Jr., O.L. 1986. Electron microscopic study of Saccharomyces cerevisiae rDNA chromatin replication. Mol. Cell Biol. 6: 1148-1157.

Sanchez, J.A., Kim, S.M., and Huberman, J.A. 1998. Ribosomal DNA replication in the fission yeast, Schizosaccharomyces pombe. Exp. Cell Res. 238: 220-230.

Shareef, M.M., King, C., Damaj, M., Badagu, R., Huang, D.W., and Kellum, R. 2001. Drosophila heterochromatin protein 1 (HP1)/origin recognition complex (ORC) protein is associated with HP1 and ORC and functions in heterochromatininduced silencing. Mol. Biol. Cell 12: 1671-1685.

Skryabin, K.G., Eldarov, M.A., Larionov, V.L., Bayev, A.A., Klootwijk, J., de Regt, V.C., Veldman, G.M., Planta, R.J., Georgiev, O.I., and Hadjiolov, A.A. 1984. Structure and function of the nontranscribed spacer regions of yeast rDNA. Nucleic Acids Res. 12: 2955-2968.

Smith, J.S. and Boeke, J.D. 1997. An unusual form of transcriptional silencing in yeast ribosomal DNA. Genes \& Dev. 11: 241-254.

Smith, J.S., Brachmann, C.B., Celic, I., Kenna, M.A., Muhammad, S., Starai, V.J., Avalos, J.L., Escalante-Semerena, J.C., Grubmeyer, C., Wolberger, C., et al. 2000. A phylogenetically conserved NAD+-dependent protein deacetylase activity in the Sir2 protein family. Proc. Natl. Acad. Sci. 97: 6658-6663.

Stevenson, J. and Gottschling, D. 1999. Telomeric chromatin modulates replication timing near chromosome ends. Genes 
\& Dev. 15: 146-151.

Stinchcomb, D.T., Struhl, K., and Davis, R.W. 1979. Isolation and characterization of a yeast chromosomal replicator. $\mathrm{Na}$ ture 282: 39-43.

Tanny, J.C., Dowd, G.J., Huang, J., Hilz, H., and Moazed, D. 1999. An enzymatic activity in the yeast Sir2 protein that is essential for gene silencing. Cell 99: 735-745.

Tham, W.H. and Zakian, V.A. 2002. Transcriptional silencing at Saccharomyces telomeres: Implications for other organisms. Oncogene 21: 512-521.

Triolo, T. and Sternglanz, R. 1996. Role of interactions between the origin recognition complex and SIR1 in transcriptional silencing. Nature 381: 251-253.

Vujcic, M., Miller, C.A., and Kowalski, D. 1999. Activation of silent replication origins at autonomously replicating sequence elements near the HML locus in budding yeast. Mol. Cell Biol. 19: 6098-6109.

Walmsley, R.M., Johnston, L.H., Williamson, D.H., and Oliver, S.G. 1984. Replicon size of yeast ribosomal DNA. Mol. Gen. Genet. 195: 260-266.

Wyrick, J.J., Aparicio, J.G., Chen, T., Barnett, J.D., Jennings, E.G., Young, R.A., Bell, S.P., and Aparicio, O.M. 2001. Genome-wide distribution of ORC and MCM proteins in $S$. cerevisiae: High-resolution mapping of replication origins. Science 294: 2357-2360. 


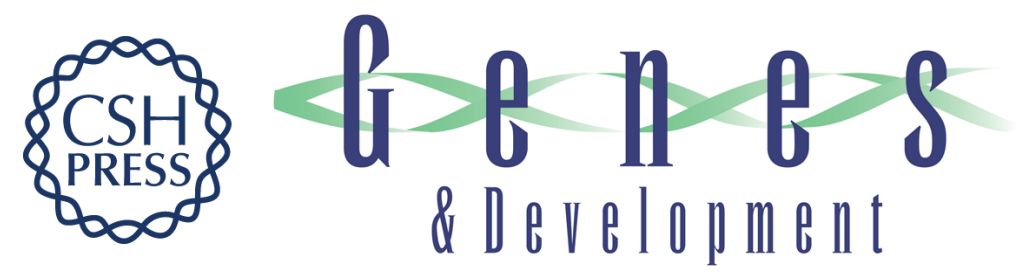

\section{To fire or not to fire: origin activation in Saccharomyces cerevisiae ribosomal DNA}

Andreas S. Ivessa and Virginia A. Zakian

Genes Dev. 2002, 16:

Access the most recent version at doi:10.1101/gad.1033702

References This article cites 48 articles, 18 of which can be accessed free at: http://genesdev.cshlp.org/content/16/19/2459.full.html\#ref-list-1

License

Email Alerting

Receive free email alerts when new articles cite this article - sign up in the box at the top Service right corner of the article or click here.

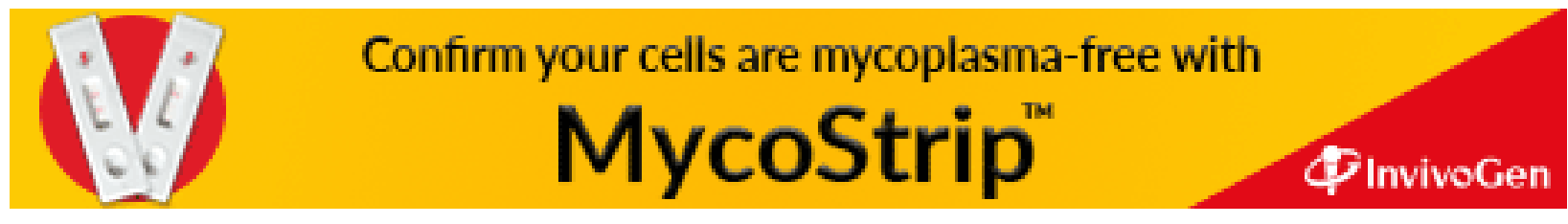

